\title{
We All Can Contribute to Training the New Generation of Pediatric Hematologists
}

To the Editor: Before commenting on Dr. Parker's reaction to our commentary we first wish to thank him for expressing them in this format. We have received numerous calls following publication of our commentary, from colleagues who concur with our concern. Dr. Parker is specifically worried that smaller pediatric hematology/ oncology programs who do not meet the criteria of seeing 60 new oncology patients per year are automatically disqualified from having a fellowship program, even if they see a large number of hematology patients. As Dr. Parker knows the Accreditation Council for Graduate Medical Education Pediatrics Residency Review Committee (RRC) is the body responsible for establishing the guidelines for training fellows and their guidelines reflect a consensus opinion of the professionals in the field. The primary responsibility of the RRC is to ensure that trainees obtain the clinical and academic skills that will transform them into bona fide subspecialists.

Programs like Dr. Parker's who are fortunate to care for a large number of hematology patients play a critical role in encouraging young colleagues to consider choosing pediatric hematology as a career. They should encourage the leadership in the Department of Pediatrics to make certain that residents spend time in the pediatric hematology clinics to gain experience in managing patients with hematological disorders. Subsequently, they should encourage residents to apply for approved fellowship programs which have a strong interest in both hematology and oncology. In addition, there is no reason why Dr. Parker's program could not play a key role in training future pediatric hematologists. It is possible for programs like his' to collaborate with the larger oncology programs to provide a unique experience in the education of hematology/oncology fellows.

Besides a meaningfully broad clinical exposure to hematology there is a need for the postgraduate fellow to gain experience in clinical design and/or laboratory research that could impact on furthering a better understanding in the management and etiology of hematological disorders. We suspect this fundamental need might be better served at larger programs that are often fortunate to have a number of investigators involved in hematological research.

In conclusion it should be our goal to provide the best training and experience possible for our postdoctoral fellows both clinically and in research. There is no reason why smaller programs cannot collaborate in training fellows with larger programs, or hematology focused ones with oncology heavy programs. All of us in the field have the ability to generate an interest in hematology care and research and encourage young colleagues to enter a field with a longstanding history of seminal contributions to medicine.

Max J. Coppes, MD, PhD, MBA* Children's National Medical Center Washington, District of Columbia

Laurence A. Boxer, MD University of Michigan School of Medicine Ann Arbor, Michigan

\footnotetext{
*Correspondence to: Max J. Coppes, Executive Director, Center for Cancer \& Blood Disorders, Children's National Medical Center, Washington, DC; Professor of Medicine, Pediatrics, and Oncology, Georgetown University, Washington, DC.

E-mail: mcoppes@cnmc.org
}

Received 9 November 2007; Accepted 9 November 2007 